\title{
Phase transition in L-alaninium oxalate by photoacoustics
}

\author{
M SIVABARATHY, S NATARAJAN, S K RAMAKRISHNAN ${ }^{\dagger}$ and K RAMACHANDRAN* \\ School of Physics, Madurai Kamaraj University, Madurai 625 021, India \\ ${ }^{\dagger}$ Department of Chemistry, Thiagarajar College, Madurai 625 009, India
}

MS received 12 April 2004; revised 4 September 2004

\begin{abstract}
Phase transition in L-alaninium oxalate is studied by using TG, DTA and photoacoustic spectroscopy. A sharp transition at $378 \mathrm{~K}$ by photoacoustics is observed whereas at the same temperature the endothermic energy change observed by TG and DTA is not very sharp. This is discussed in detail with reference to the other known data for the organic crystals.
\end{abstract}

Keywords. L-alaninium oxalate; phase transition; DSC; TG and DTA; photoacoustics.

\section{Introduction}

Amino acids are the fundamental units of proteins and hence general reports on such systems, particularly in the understanding of crystal structures are available in the literature. Due to the limited experimental facilities, studies on many of the structural transitions using XRD could not be carried out and so alternative techniques are being used in the study of phase transitions. Photo pyroelectrics and photoacoustics are some of the efficient alternative techniques to study phase transitions, particularly for the systems that are highly absorbing. Recently, glycine phosphite (GPI) single crystals were studied for ferroelectric phase transition by photo pyroelectric technique by monitoring the thermal parameters (Preethi Menon and Philip 2001). The order-disorder type transition is observed at $224 \mathrm{~K}$ in this crystal. It is known that GPI is the second hydrogen-bonded ferroelectric crystal of an amino acid with phosphorous acid, after betanine phosphite.

\section{Experimental}

Recently, in our X-ray laboratory, another amino acid complex, viz. L-alaninium oxalate (LAO) was grown and the crystal structure elucidated (Subha Nandhini et al 2001). These crystals were grown from a saturated aqueous solution containing L-alanine and oxalic acid in equimolar ratio. The crystals appeared in the form of colourless needles of size, $\sim 10 \times 5 \times 2 \mathrm{~mm}$.

\subsection{Crystal structure}

The crystals of LAO belong to the orthorhombic system and has the unit cell dimensions: $a=5 \cdot 630(2) \AA, b=$

*Author for correspondence
$7 \cdot 235(2) \AA, c=19 \cdot 597(3) \AA$, the space group being $P 2{ }_{1} 2_{1} 2_{1}$ and has four molecules in the unit cell.

The amino group of the L-alaninium cation forms three $\mathrm{N}-\mathrm{H}$... O hydrogen bonds with the oxygens of semi oxalate group and a symmetry-related alanine cation. There also exists two O-H...O hydrogen bonds. The alaninium and semi-oxalate ions form alternate columns leading to a layered arrangement parallel to the a.c. plane and each such layer is interconnected to the other through $\mathrm{N}-\mathrm{H}$...O hydrogen bonds. Two short C...O contacts involving the carboxyl oxygen of the alaninium ion $[\mathrm{C} 1 \ldots \mathrm{O} 2(1 / 2+x$, $3 / 2-y,-z)=2 \cdot 931(3) \AA$ and $\mathrm{C} 2 \ldots \mathrm{O} 2(-1 / 2+x, 3 / 2-y$, $-\mathrm{z})=2 \cdot 977(3) \AA]$ are also observed in these layers. The slight difference observed in the bond lengths of C5O5 $[=1 \cdot 219(2) \AA]$ and C5-O6 $[=1 \cdot 235(2) \AA]$ in the carboxylate group of the semi-oxalate ion may be attributed to the difference in strengths of the $\mathrm{N}-\mathrm{H}$... O hydrogen bonds in which both $\mathrm{O} 5$ and $\mathrm{O} 6$ are involved (table 1).

This bond strength, in general, will be given in terms of the activation and formation energies. For example, it is found to be $17 \mathrm{~kJ} / \mathrm{mole}$ in glycine phosphite. When the temperature of the system is varied, it is expected that the dynamics of the bond with low strength would weaken and subsequently order-disorder transition should creep in. Since in LAO, C5-O6 is weak compared to C5-O5, this will be prone to changes abruptly thus leading to phase transitions when temperature is increased.

Here, we report a detailed study of the variation of thermal parameters of this L-alaninium oxalate, measured along the principal directions, for various temperatures employing a photoacoustic method. TG and DTA measurements were first carried out to identify any transitions. The calorimetric techniques such as DSC, TG and DTA are generally used to study the phase transition. But, Ganguly and Rao (1981) reported from their PA study on $\mathrm{La}_{2} \mathrm{CuO}_{4}$ that there would be a weak transition at $553 \mathrm{~K}$ but they could not see this in DSC and DTA i.e. weak 
Table 1. Hydrogen bonding scheme for LAO.

\begin{tabular}{|c|c|c|c|c|}
\hline D-H...A & D-H $(\AA)$ & H...A $(\AA)$ & D...A $(\AA)$ & $\angle \mathrm{D}-\mathrm{H} \ldots \mathrm{A}\left(^{\circ}\right)$ \\
\hline N1-H1A...O $6^{\mathrm{i}}$ & $0 \cdot 89$ & 1.91 & $2 \cdot 728(2)$ & 152 \\
\hline $\mathrm{N} 1-\mathrm{H} 1 \mathrm{~B} \ldots \mathrm{O} 4^{\mathrm{ii}}$ & 0.89 & $2 \cdot 28$ & $3 \cdot 085(2)$ & 150 \\
\hline $\mathrm{N} 1-\mathrm{H} 1 \mathrm{C} \ldots \mathrm{O} 2^{\mathrm{iii}}$ & $0 \cdot 89$ & $2 \cdot 30$ & $2.978(2)$ & 133 \\
\hline N1-H1C...O5 & $0 \cdot 89$ & $2 \cdot 35$ & $2 \cdot 896(2)$ & 120 \\
\hline $\mathrm{O} 1-\mathrm{H} 1 \ldots \mathrm{O} 5^{\mathrm{iv}}$ & $0 \cdot 82$ & 1.76 & $2 \cdot 575(2)$ & 170 \\
\hline $\mathrm{O} 3-\mathrm{H} 3 \ldots \mathrm{O}^{\mathrm{v}}$ & $0 \cdot 82$ & 1.73 & $2 \cdot 545(2)$ & 172 \\
\hline \multicolumn{5}{|l|}{ Short contacts } \\
\hline $\mathrm{C} 1 \ldots \mathrm{O} 2^{\mathrm{iv}}$ & & & $2 \cdot 931(3)$ & \\
\hline $\mathrm{C} 2 \ldots \mathrm{O} 2^{\mathrm{iv}}$ & & & $2 \cdot 977(3)$ & \\
\hline
\end{tabular}

Symmetry codes: (i) $-x, 1 / 2+y, 1 / 2-z$; (ii) $1-x, 1 / 2+y, 1 / 2-z$; (iii) $x-$ $1 / 2,1 / 2-y,-z$; (iv) $1 / 2+x, 1 / 2-y,-z$; and (v) $1+x, y, z$.

transitions can be effectively seen in PA technique compared to any other conventional techniques.

\subsection{TG and DTA}

These measurements were carried out from ambient temperature to $400^{\circ} \mathrm{C}$ in steps of $10^{\circ} \mathrm{C}$ using the instrument STA 409C (NETZCH-Geratebau GmbH thermal analyser) for LAO with alumina as the reference material. Correlation was clearly monitored with this and the result is shown in figure 1. During the endothermic reaction, at around $102^{\circ} \mathrm{C}$, DTA reaches a maximum and starts decreasing. Similarly, at $209.8^{\circ} \mathrm{C}$ there is another transition. Eventhough this one at $209 \cdot 8^{\circ} \mathrm{C}$ is more pronounced than that at $102^{\circ} \mathrm{C}$, the weak transition at $102^{\circ} \mathrm{C}$ should be verified by some other alternative techniques.

So, photoacoustic study on L-alaninium oxalate is carried out as there are only weak interactions.

\subsection{Photoacoustic spectroscopy (PAS)}

It is known that when an intermittent light is absorbed by a crystalline sample kept in an air-tight cell, the internal energy increases and hence thermal waves are generated. This heat energy diffuses into the nearby air medium causing an increase or decrease in the pressure (due to intermittent light) inside the PA cell. This is the acoustic wave and is detected by a microphone attached close to the sample.

Pichon, as early as in 1979, had used PAS successfully to study the phase transition in solids. It is known that for thermally thick samples the PA signal is proportional to

$$
\frac{\mu_{g}}{T_{0}}\left(\frac{2}{\rho_{\mathrm{s}} C_{\mathrm{s}}}\right)
$$

where $C_{\mathrm{s}}$ is the specific heat, $\mu_{\mathrm{g}}$ thermal diffusion and $\rho_{\mathrm{s}}$ the density of the solid. Even though the signal seems to

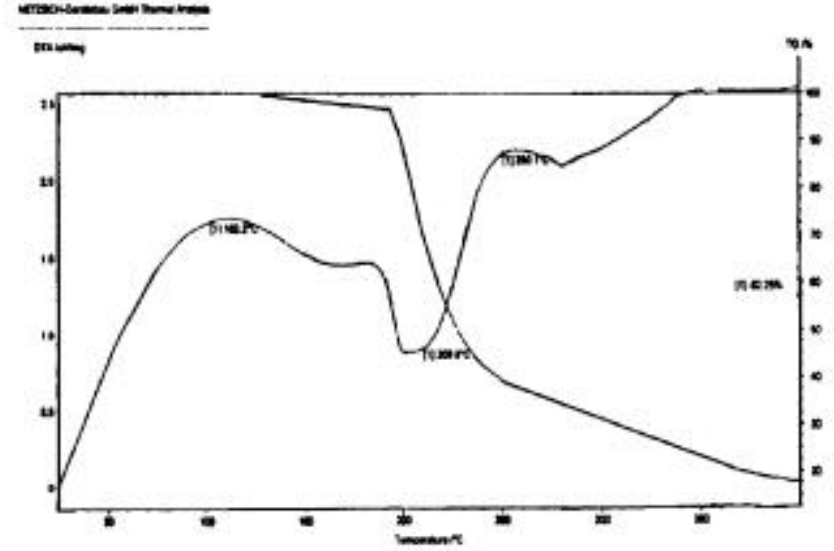

Figure 1. TG and DTA for L-alaninium oxalate.

vary as $1 / T_{0}$, in most cases it is found that while heating a fresh sample, the PA signal increases with temperature and reaches a maximum before it starts decreasing in the form of $1 / T$. This is associated with the presence of large absorbed or condensed phase of the sample. But if there is any transition from one phase to another, one finds an abrupt change in the PA signal rather than the $1 / T$ variation.

2.3a Photoacoustic measurements: The present PA spectrometer has xenon lamp $400 \mathrm{~W}$ (Juon Youn), monochromator (Juon Youn), PAR chopper, DSP 7225 lockin amplifier (PAR), $20 \mathrm{MHz}$ GOULD digital storage oscilloscope (DSO), a stainless steel PA cell and a condenser microphone.

For the photoacoustic measurements, the single crystal itself can as well be used when depth profiling (variation of the PA signal with the chopping frequency for a particular wavelength of the incident light) alone is carried out. So for this part of the experiment, the monochromator was removed and straightaway the xenon lamp was used. The sample, LAO, with a thickness of about $500 \mu \mathrm{m}$ is mounted in the PA cell, in the preferred orientation $(b$ 
axis). The PA signal was first observed on the DSO and then the measurements were made using the lockin amplifier. The experiment is also repeated with the powdered LAO i.e. the sample is ground to powder and pelletized by applying suitable hydrostatic pressure. This pellet is then used for PA measurements.

The sample is then heated to various temperatures. This is done as follows. The PA cell was wound with 32 gauge Nichrome wire externally, suitably pasted between the layers. This is then connected to a variac which was quite stable. A thermocouple was placed near the sample inside the PA cell and connected to a digital thermometer thereby the temperature of the sample is monitored.

The chopper is fixed at a frequency of $22 \mathrm{~Hz}$ to start with. This is fixed from the experience for the characteristic frequency on the LAO at room temperature. The temperature is then varied in steps of $2^{\circ} \mathrm{C}$ and the corresponding PA signal is observed as given in figure 2 . The same measurements were repeated for three different chopping frequencies and every time the trend of the graph was same and so not given. This figure clearly shows that there is an abrupt change in the PA amplitude at a temperature of $101^{\circ} \mathrm{C}$. The rate of change is so fast that it is around $16 \mathrm{mV} /{ }^{\circ} \mathrm{C}$. Such a rapid change in the PA amplitudes clearly indicates that there is a phase transition. This is in contrast with the TG and DTA measurements where the variation is not very rapid which is only $1 \mathrm{mV} /$ $25^{\circ} \mathrm{C}$ i.e. we cannot exactly pinpoint the transition temperature in TG and DTA whereas PA measurements sharply determine the transition point. This PA measurement was repeated twice and the same result could be reproduced.

In order to ascertain this transition, the same measurements were repeated when the sample was cooled from $150^{\circ} \mathrm{C}$. Those measurements corresponding to the cooling

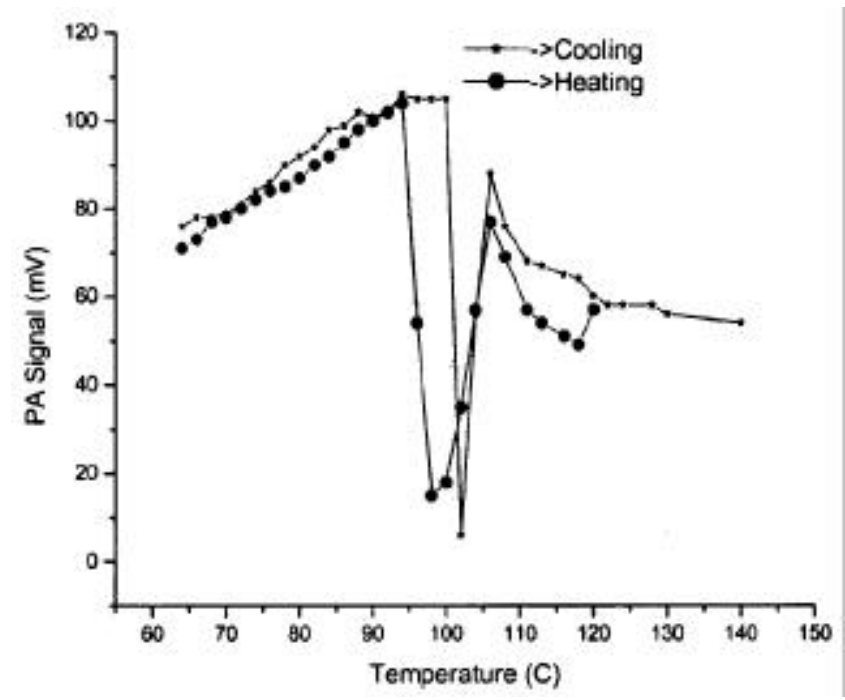

Figure 2. Variation of PA signal with temperature for L-alaninium oxalate. cycle, is given in the same figure 2 . The transition here is observed at $98^{\circ} \mathrm{C}$.

\section{Discussion}

Phase transitions between polymorphs are very common in organic crystals and the mechanisms remain unknown despite the large amount of studies. No such phase transition studies were reported in the literature for LAO, even though results are available for other organic crystals. Recently, Hashizume et al (2003) observed the phase transition of L-ethyl-3-urea by means of detailed temperature resolved single crystal diffraction method. Crystal structures before and after the phase transition are isostructural where one-dimensional hydrogen bonding structure is formed and stacked to form a molecular layer. This work showed a solid to solid phase transition at $363 \mathrm{~K}\left(90^{\circ} \mathrm{C}\right)$. Since these crystals were grown by slow evaporation method, they have taken care in omitting the occluded water, if any, during the growth by annealing the sample fairly at higher temperature of $378 \mathrm{~K}$ for about an hour (the melting point of this system is $381 \mathrm{~K}$ ). Also the measurements were carried out for both heating and cooling cycles. During heating, the transition is observed at $363 \mathrm{~K}$ whereas the same in cooling cycle is $368 \mathrm{~K}$.

Now, the present results on LAO show a phase transition (solid to solid) at $374 \mathrm{~K}\left(101^{\circ} \mathrm{C}\right)$, when the temperature is slowly increased from room temperature, which is of the same order as for L-ethyl-3-urea. Here also, we have followed the same precautions as Hazhizume et al (2003). To start with, the sample of LAO was annealed at $150^{\circ} \mathrm{C}$ in air for about $2 \mathrm{~h}$, thereby the chance of getting the water molecule inside the crystal is ruled out. Then the PA measurements were recorded for this sample. Similarly, after reaching $150^{\circ} \mathrm{C}$, the sample was allowed to cool down to room temperature. For every $5^{\circ} \mathrm{C}$ fall the PA signal was observed and the graph was plotted on the same (figure 2). The comparison with L-ethyl-3-urea is made here because both of them have layered structures. The layer structure of the present LAO, viewed from the $b$ axis is shown in figure 3 .

Even though the geometry of the layer is retained, the relative position of the layer in LAO with its neighbours changes gradually with temperature. The change is accelerated at the temperature representing the start of the endotherm in the TG and DTA curves. The structural variation thus creates a void space in between the layers and as this grows then naturally the crystal will be unstable and so the carboxyl group on the last of the molecules turns into a disordered structure with abrupt conformational changes to fill up the void space. This is confirmed from the photoacoustic measurements also. That is when defects (voids) are more, the PA signal will be strong and here we find at $102^{\circ} \mathrm{C}$ (at $375 \mathrm{~K}$ ), there is a steep variation supporting the TG and DTA results of large void space 
between the layers at $102^{\circ} \mathrm{C}$. This transition, being isostructural, may be visualized as the transition of two elementary processes - supramolecular and molecular.

During the cooling cycle, the transition is observed at $98^{\circ} \mathrm{C}$. This proves again that we have not reached the melting point when the measurements are done (The melting point is $188^{\circ} \mathrm{C}$ ).

The present PA study on LAO is also useful as the phase transition study on single crystals by XRD, especially for first-order phase transition, causes the collapse of the single crystal under study and so this is restricted to only high-order phase transitions. So, for crystals having laye-

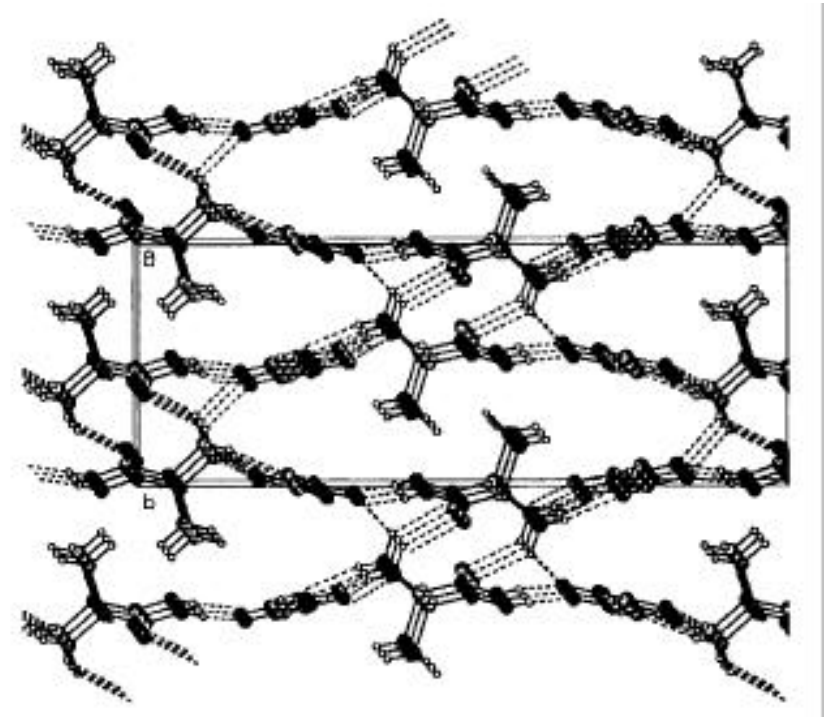

Figure 3. Layered structure of L-alaninium oxalate. red structures, photoacoustic study on phase transition will give a wealth of information compared to XRD or TG and DTA. Also, it is once again proved that PA study on phase transitions will be very much reliable than other calorimetric techniques, DSC, TG and DTA.

Very interesting results are now reported by Dhanuskodi and Vasantha (2004) on this LAO for phase transition. They have carried out structural, thermal and optical characterization for this system to report that there is no phase transition in this LAO crystal. There is no specific experiment carried out by these authors for the phase transition except TG/DTA that too with a larger scan of $20^{\circ} \mathrm{C} / \mathrm{min}$. We have repeatedly mentioned here that TG/ DTA alone will not be enough for phase transition and this had been demonstrated by Ganguly and Rao as early as in 1981. Also they have not carried out the annealing process on this solution grown sample and still they have not seen anything near the boiling point of water in the endothermic curve. There is every possibility for these authors to skip the crucial point on phase transition. Another ambiguous result is the melting point of the system from the TG/DTA appears to be around $203^{\circ} \mathrm{C}$ whereas they have the same from the melting point apparatus as $188^{\circ} \mathrm{C}$. It is to be checked whether the system quoted is LAO or a derivative of LAO.

Due to this result, we have carried out another measurement on TG/DTA for the annealed sample LAO. This is essential as the original TG/DTA is only for the as grown sample. The scan rate is further reduced to $5^{\circ} \mathrm{C}$ and the new endotherm clearly shows a dip at $98^{\circ} \mathrm{C}$. This is now given in figure 4.

So, we finally report that there should be a phase transition at about $98^{\circ} \mathrm{C}$ in this L-alaninium oxalate

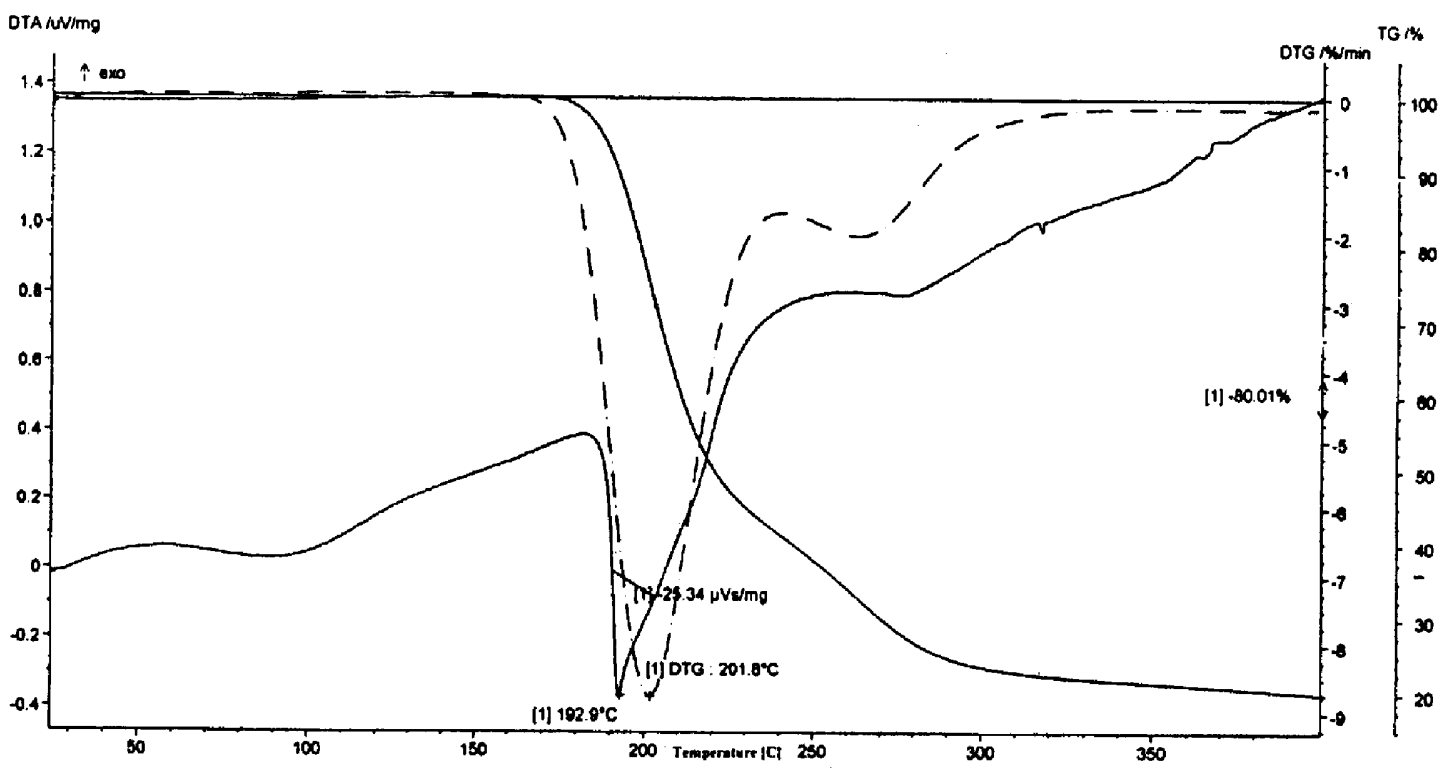

Figure 4. TG and DTA for L-alaninium oxalate after annealing. 
and photoacoustics is the best tool for this detection apart from the XRD.

\section{Acknowledgement}

One of the authors (KR) acknowledges the University Grants Commission (DRS \& COSIST), New Delhi, for financial support in setting up a photoacoustics laboratory.

\section{References}

Dhanuskodi S and Vasantha K 2004 Cryst. Res.Technol. 39259 Ganguly P and Rao C N R 1981 Proc. Indian Acad. Sci. (Chem. Sci.) 90153

Hashizhume D et al 2003 Acta Crystallogr. B59 404

Pichon C 1979 Appl. Phys. Lett. 351435

Preethi Menon C and Philip J 2001 Mater. Res. Bull. 362407

Subha Nandhini M, Krishnakumar R V and Natarajan S 2001 Acta Crystallogr. $\mathbf{E 5 7}$ o633 